\title{
Significance and utility of MR cisternography for diagnosis and treatment of trigeminal neuralgia ${ }^{*}$
}

\author{
Tatsurou Tanaka ${ }^{1}$, Shunji Shiiba ${ }^{2}$, Eiji Sakamoto ${ }^{3}$, Masafumi Oda $^{1}$, Shinji Kito $^{1}$, Kentaro Ono $^{4}$, \\ Nao Wakasugi-Sato ${ }^{1}$, Shinobu Matsumoto-Takeda', Yuji Seta ${ }^{5}$, Yoshiki Imamura, \\ Seiji Watanabe ${ }^{2}$, Kiyotoshi Inenaga ${ }^{4}$, Yasuhiro Morimoto ${ }^{1,7 \dagger}$ \\ ${ }^{1}$ Division of Diagnostic Radiology, Kyushu Dental University, Kitakyushu, Japan \\ ${ }^{2}$ Division of Dental Anesthesiology, Kyushu Dental University, Kitakyushu, Japan \\ ${ }^{3}$ Department of Control of Physical Functions, Faculty of Dentistry, Kyushu University, Fukuoka, Japan \\ ${ }^{4}$ Division of Physiology, Kyushu Dental University, Kitakyushu, Japan \\ ${ }^{5}$ Division of Oral Histology and Neurobiology, Kyushu Dental University, Kitakyushu, Japan \\ ${ }^{6}$ Department of Oral Diagnostic Science, Nihon University School of Dentistry, Tokyo, Japan \\ ${ }^{7}$ Center for Oral Biological Research, Kyushu Dental University, Kitakyushu, Japan \\ Email: ${ }^{\dagger}$ rad-mori@kyu-dent.ac.jp
}

Received 16 January 2013; revised 24 February 2013; accepted 5 March 2013

\begin{abstract}
This review article provides doctors and dentists with an introduction to the clinical applications and significance of magnetic resonance cisternography (MRC) for the evaluation of neurovascular compression of the trigeminal nerve in patients with trigeminal neuralgia (TN). Specifically, the method and characteristics of MRC are first explained using the relevant MR sequences. Next, clinical applications for the diagnosis of TN are discussed. Finally, the significance and utility of MRC for effective treatment of patients with TN, such as carbamazepine administration and/or local anesthetic blocks, are presented.
\end{abstract}

Keywords: Trigeminal Neuralgia; Diagnosis; MR Cisternography; Carbamazepine; Local Anesthetic Block

\section{INTRODUCTION}

Trigeminal neuralgia (TN) is an important disease characterized by severe lancinating pain in the oral and maxillofacial regions. A common cause of TN is neurovascular compression (NVC) in the root entry zone (REZ) of the trigeminal nerve in the cerebellopontine angle cistern [1-3]. For the detection of NVC in the REZ of the trigeminal nerve using magnetic resonance imaging (MRI), two methods have been reported: magnetic resonance angiography (MRA) [4-6] and magnetic resonance

\footnotetext{
${ }^{*}$ Conflict of interest statement: no potential conflicts of interest were disclosed.

\#This study was supported in part by grants-in-aid for scientific research from the Ministry of Education, Science, Sports and Culture of Japan, and from Kitakyushu City to YM.

${ }^{\dagger}$ Corresponding author.
}

cisternography (MRC) [7-12]. It has been speculated that neurovascular structures in the REZ of the trigeminal nerve are shown with better resolution by MRC than by MRA [7,9-12]. Presently, as one of the tools to visualize NVC in the REZ, three-dimensional (3D)-MRC has been clinically applied for patients with TN. MRC is easy to acquire by simply obtaining additional sequences without any invasivity to patients. It takes a few minutes for the acquisition of MRC. In our dental hospital, MRC is required for all patients with TN from suspected central nervous system causes. To our knowledge, there has been no review article on the significance and utility of MRC for patients with TN; therefore, many doctors and dentists might not be aware of this diagnostic and therapeutic modality. Additionally, the methods of MRC for patients with TN are not standardized. Therefore, the goals of this review are to introduce the clinical applications of MRC for patients with TN as used in our dental hospital, and then to discuss the methods and applications of MRC $[7,8,13]$. At the practical level, the sequences and characteristics of MRC will first be detailed, followed by the clinical applications. Next, the identification of NVC of the trigeminal nerve in the cerebellopontine angle cistern using MRC and its clinical significance will be described, drawing upon discussions from our previous reports $[7,8,13]$. At the same time, we will demonstrate how the volume of NVC, as measured by MRC, can predict the efficacy of carbamazepine (CBZ) therapy as one of the initial treatments for patients with TN. Finally, the relationship between NVC volume measured by MRC and the success of local anesthetic block by tetracaine (TNB) will be described in patients with TN for whom CBZ therapy was indicated but less likely to offer relief [14,15]. 


\section{DESCRIPTION OF MRC: ITS METHODS AND CHARACTERISTICS USING RELEVANT MR SEQUENCES}

MRC uses hydrography for visualizing nerves and blood vessels in the cerebrospinal fluid. The use of MRC allows precise visualization of the trigeminal nerve running through the REZ (Figure 1). It follows that MRC can evaluate NVC of the trigeminal nerve in patients with TN (Figure 2). Commonly, constructive interference with steady state (CISS), fast imaging with steady precession (FISP), and fast asymmetric spin echo (FASE) should be applied as sequences of MRC. Basically, there is no difference between the visualizations of MRC among these three sequences. It has been reported that the quality of MRC with 3D-FASE is significantly higher than that with 3D-CISS as the result of differences in flow and susceptibility to magnetic artifact [16]. A FASE sequence, as mentioned above, produces a heavy T2-weighted image that has excellent resolution because the section thickness can be decreased to as little as 0.8 $\mathrm{mm}$ and the voxel size can be decreased to as little as 0.6 $\mathrm{mm}^{3}$, with a short acquisition time using 3D-excitation [16-21]. MR imaging with 3D-FASE has been established as a useful tool for MR cholangiopancreatography [22-24], MRC [16-19], and MR sialography [20,21].

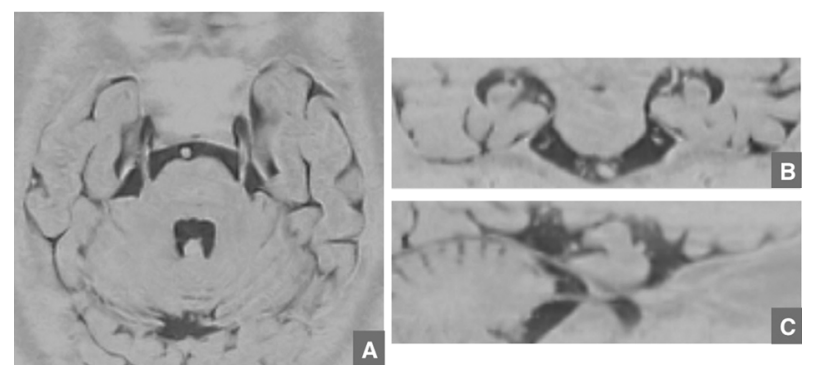

Figure 1. MR images of the trigeminal nerve in the REZ seen on MRC with 3D-FASE of a normal 50-year-old male. Axial (A), reformatted coronal (B), and reformatted sagittal (C) images can precisely visualize the right trigeminal nerve running in the REZ.

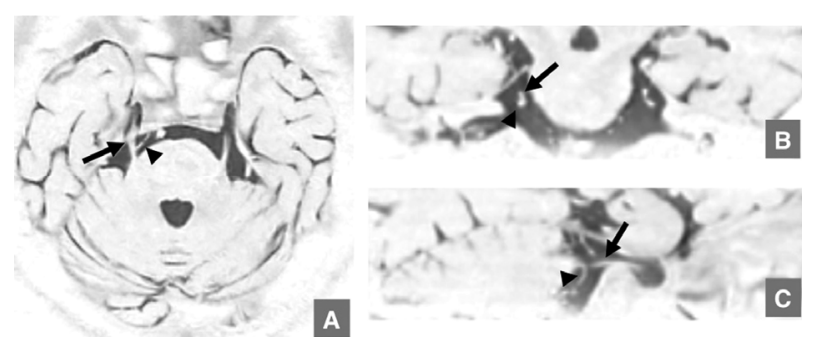

Figure 2. MR images of the trigeminal nerve (arrows) in the REZ seen on MRC with 3D-FASE of a 57-year-old female with TN caused by NVC in the superior cerebellar artery (arrowheads). Axial (A), reformatted coronal (B), and reformatted sagittal (C) images can precisely visualize the NVC of the right trigeminal nerve running in the REZ.
There have been some reports that MRC, with 3D-FASE in particular, is useful for the detection of brain tumors and NVC in the cerebellopontine angle [16-19]. Our hospital is equipped with a 1.5-T full-body MR system (EXCELART Vantage $^{\mathrm{TM}}$ powered by Atlas; Toshiba, Tokyo, Japan), and only MRC using the FASE sequence can be acquired. Therefore, we introduced the clinical applications of MRC using FASE for patients with TN in our hospital.

First, we will explain the methods of MR examination for patients with TN. For patients with TN, T1-weighted, T2-weighted, and MRC images are acquired for determining whether NVC (Figure 1) and space-occupying lesions (SOL) can be clearly identified. In particular, T1and T2-weighted images are obtained to rule out SOL such as brain tumors (Figure 3 ) and cerebral infarctions. The imaging parameters used in the two MR methods are shown in Table 1. MRC via 3D-FASE is acquired using the parameters listed in Table 2 [7,8]. Conventional sagittal, coronal, and transverse scout MR images of the head are initially obtained. The acquisition slab is oriented in the transverse direction on the sagittal and coronal scout images so that the trigeminal nerve on both sides can be included in the image. After data acquisition is completed and 50 sections are obtained, coronal and sagittal reformatted images are obtained by using a multi-planar reconstruction (MPR) algorithm. In addition, images with inversion between black and white are used
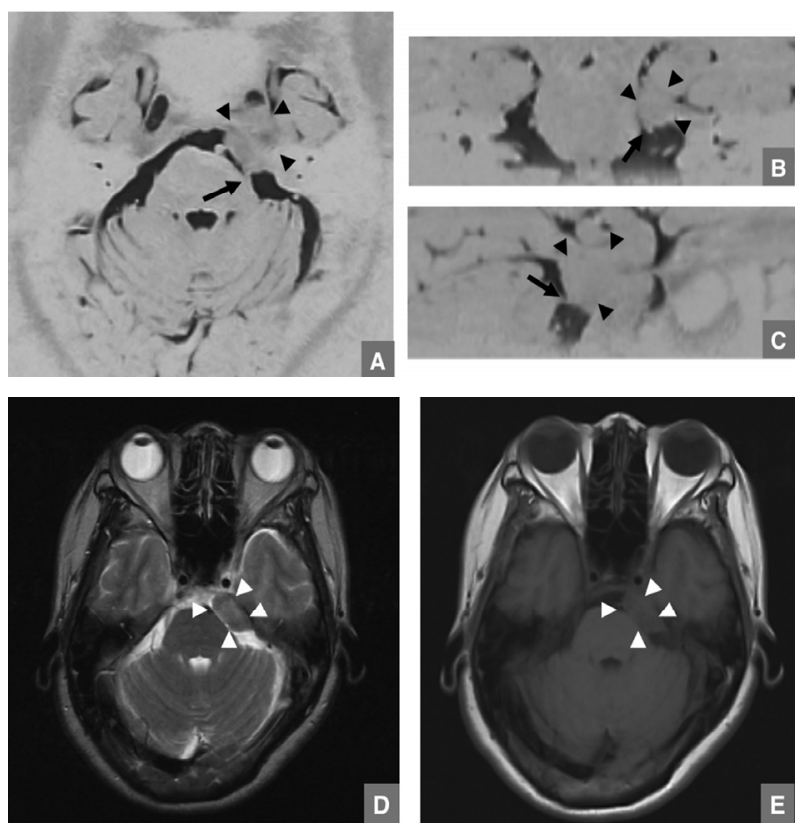

Figure 3. MR images of the trigeminal nerve (arrows) in the REZ seen on MRC with 3D-FASE of a 57-year-old female with TN. Axial (A), reformatted coronal (B), reformatted sagittal (C) images by MRC with 3D-FASE, and T2-weighted (D) and T1-weighted (E) images can visualize that the brain tumor (arrowheads) invaded the REZ region of the trigeminal nerve. 
Table 1. Imaging parameters.

\begin{tabular}{ccc}
\hline & \multicolumn{2}{c}{ Sequences } \\
\cline { 2 - 3 } & T1WI & T2WI \\
\hline TR (ms) & 500 & 4000 \\
TE (ms) & 15 & 100 \\
Flip angle ( $\left.{ }^{\circ}\right)$ & 90 & 90 \\
FOV (mm) & $220 \times 220$ & $220 \times 220$ \\
Section thickness (mm) & 6.0 & 6.0 \\
Intersection gap & 1.0 & 1.0 \\
Slab thickness (mm) & 119 & 119 \\
Matrix (pixels) & $256 \times 256$ & $256 \times 256$ \\
\hline
\end{tabular}

TR, repetition time; TE, echo time; FOV, field of view.

Table 2. Parameters of MR cisternography.

\begin{tabular}{cc}
\hline TR $(\mathrm{ms})$ & 6000 \\
TE $(\mathrm{ms})$ & 250 \\
Flip angle $\left(^{\circ}\right)$ & 90 \\
FOV $(\mathrm{mm})$ & $150 \times 150$ \\
Section thickness (mm) & 1.0 \\
Intersection gap & 0 \\
Slab thickness (mm) & 50 \\
Matrix (pixels) & $192 \times 192$ \\
\hline
\end{tabular}

MR, magnetic resonance; TR, repetition time; TE, echo time; FOV, field of view.

from MRC with 3D-FASE to evaluate NVC (Figures 1 and 2) $[7,8,13,16,25-28]$. The other two reformatted images, angled parallel and perpendicular to the trigeminal nerve at the REZ (Figures 1-3), are also obtained by using a MPR algorithm. For the interpretation of MR images, both the original transverse images and the four reformatted images are reviewed.

Next, radiologists use MRC to evaluate NVC by two methods. First, images from the MR methods are evaluated in detail for these characteristics: the presence of vascular contact with the trigeminal nerve at the REZ; identification of the site of NVC in the REZ of the trigeminal nerve; and the NVC volume. NVC is diagnosed when contact between the blood vessel and the nerve at the REZ is clearly detected in two or more sections of transverse images, the coronal and sagittal reformatted images, and the other reformatted images obtained parallel or perpendicular to the trigeminal nerve at the REZ on MRC (Figures 1-3). The site of the blood vessel compressing the nerve is classified as medial, lateral, cranial, or caudal according to Yoshino et al. [9]. The second method of evaluating NVC is by measuring

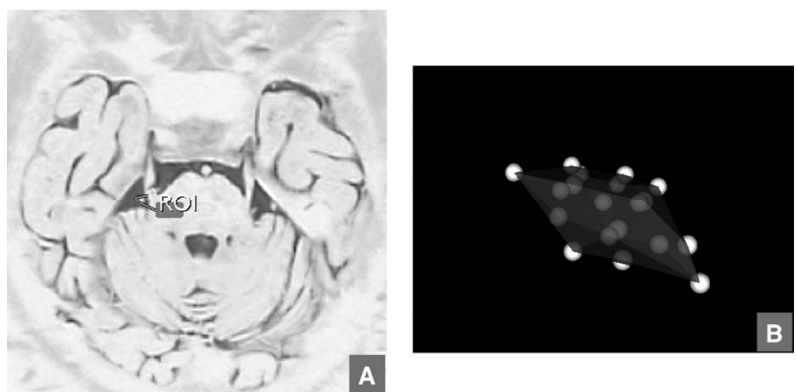

Figure 4. The photographs (A) and visual images (B) are shown when NVC volume on MRC is measured using OsiriX volume calculation software.

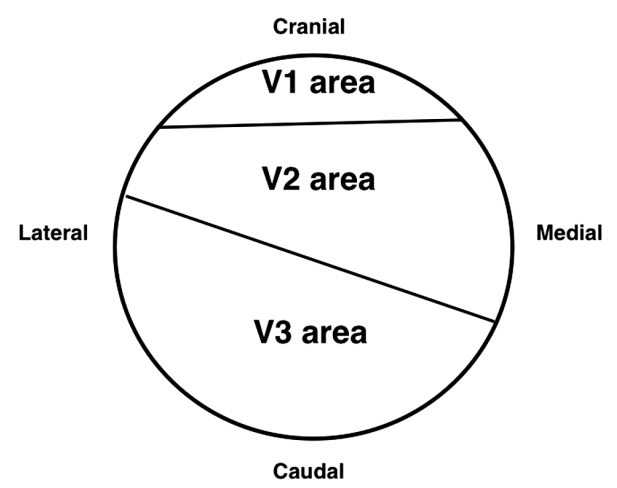

Figure 5. Scheme demonstrating the general correlation between the classification of NVC sites and the trigeminal nerve fiber array at the REZ on cross-section.

its volume, as follows [7]: the MRC images are sent to a workstation (Mac Pro; Apple Corp., Cupertino, CA, USA), and the NVC volume on the images is measured using open-source OsiriX medical imaging software (http://www.osirix-viewer.com) (Figure 4). Each NVC site is judged to correspond to each branch on the basis of the detailed anatomy of the intracranial portion of the trigeminal nerve according to Gudmundsson et al. (Figure 5) [29].

\section{CLINICAL APPLICATIONS OF MRC FOR EVALUATION OF NVC IN PATIENTS WITH TN}

Three dimensional (3D)-MRC is a useful tool for the detection of NVC in the REZ of the trigeminal nerve because of having better resolution for the detection of NVC $[4-6,9,10]$. In fact, the presence and site of NVC in the trigeminal nerve have been seen to be correctly detected by MRC with 3D-FASE in some patients who underwent microvascular decompression surgery [7]. Even the status of a trigeminal nerve surrounded with a mesh of blood vessels was precisely shown on MRC with 3D-FASE (Figure 6) [7]. Very recently, visualization of NVC in the REZ using 3D-MRC has been used as 

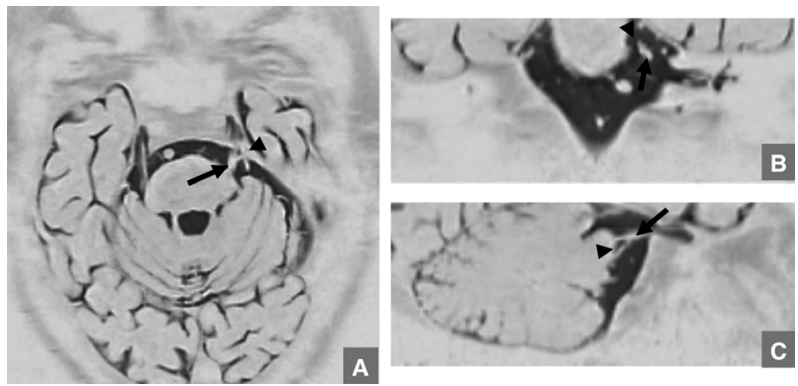

Figure 6. MR images of the trigeminal nerve (arrows) in the REZ seen on MRC with 3D-FASE of a 70-year-old male with TN caused by NVC in the superior cerebellar artery (arrowheads). Axial (A), reformatted coronal (B), and reformatted sagittal (C) images can precisely visualize the status of the trigeminal nerve surrounded with a mesh of blood vessels.

a method of preoperative simulation and as an intra-operative surgical guide for NVC in patients with idiopathic trigeminal neuralgia because of the improved accuracy of 3D-MRC [11,12,17].

In our previous study using MRC with 3D-FASE sequences, NVC was found in the symptomatic sides of 89 of the 150 patients with TN who were evaluated [7]. At the same time, a very close relationship was found between the site of NVC in the REZ as seen on 3D-MRC and the clinical neuralgic manifestations in patients with TN [7-10]. Therefore, NVC should clearly be considered as one of the causes of $\mathrm{TN}$, and we concluded that 3D-MRC was a useful tool for the detection of NVC in the REZ of the trigeminal nerve. However some patients with NVC of the trigeminal nerve, the NVC sites identified on MRC did not match the clinically manifested regions of the branches of the trigeminal nerve. It might be difficult for us to judge the NVC site in the REZ of the trigeminal nerve in cases changing over from medial to cranial or from lateral to caudal.

To our knowledge, it has not yet been reported how many subjects who do not have TN may have NVC. However, the ratio of NVC in the asymptomatic side could help to answer the question. In our previous report using MRC with 3D-FASE sequences, NVC was found in the asymptomatic sides of 27 (18\%) patients out of the 150 study subjects with TN whom we evaluated [7]. A significant difference between the sides with or without TN on the ratio of NVC was revealed. Our study results show that radiologists should consider $\mathrm{TN}$ as a possible diagnosis based on the identification of NVC using MRC.

\section{CLINICAL SIGNIFICANCE OF MRC IN PREDICTING THE CURATIVE EFFICACY OF CBZ FOR PATIENTS WITH TN}

We have found that none of the following patient-related factors play any significant role in the degree of TN, including the degree of pain and the prognosis after various types of treatment: sex, patient age, history of systemic hypertension, state of neuralgia including NVC, duration of neuralgia, or history of failed trigeminal surgery [30]. Because the extent of NVC of the trigeminal nerve can be measured by MRC, the relationship between the degree of NVC of the trigeminal nerve and the extent of pain or the curative efficacy of CBZ administration as initial treatment for patients with $\mathrm{TN}$ can be elucidated.

The method for measuring the relationship between the presence of NVC and pain has been direct observation during the operation for eliminating NVC, but the degree of pain in patients with TN having NVC could not be inferred [30,31]. Using 3D-MRC, the degree of pain in patients with TN having NVC could not be predicted $[7,8]$. In addition, our previous report also demonstrated that some patients without TN had NVC in the REZ, as seen on MRC (Figure 7) [7]. Therefore, it should be concluded that there is no relationship between the degree of NVC of the trigeminal nerve and the extent of pain. One possible explanation is that the pain caused by NVC may be related not only to the quantity of NVC in the trigeminal nerve, but also to other factors including a patient's age and sex. In particular, the degree of pain in TN may greatly depend on a patient's own sense of pain.

However, a significant correlation has been reported between NVC volume, as shown by MRC, and the curative efficacy of CBZ at $100 \mathrm{mg} /$ day for 2 weeks as an initial treatment for patients with TN [8]. This is commonly the initial treatment for patients with $\mathrm{TN}$, and is the practice in our dental hospital. If improvement is not apparent after initial treatment with CBZ at $100 \mathrm{mg} /$ day, then additional CBZ doses may be administered [32,33]. The significance of MRC for the prediction of the prognosis after initial treatment with CBZ in patients having TN with NVC should be proved. Meanwhile, physicians

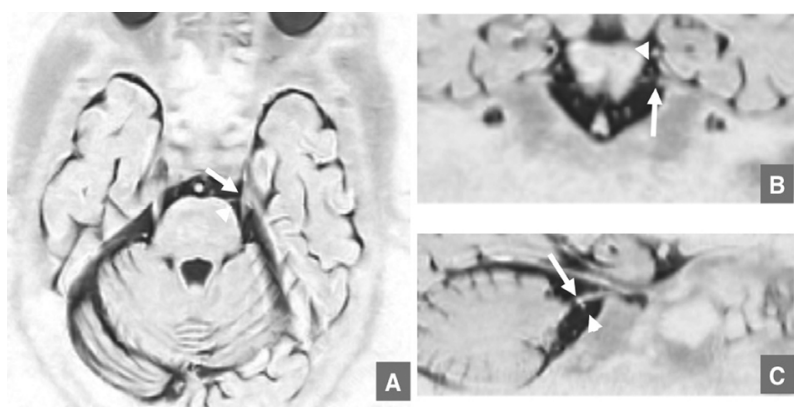

Figure 7. MR images of the trigeminal nerve (arrows) in the REZ seen on MRC with 3D-FASE of a 76-year-old male without TN caused by NVC in the superior cerebellar artery (arrowheads). Axial (A), reformatted coronal (B), and reformatted sagittal (C) images can precisely visualize NVC of the right trigeminal nerve running in the REZ. 
can confidently explain the utility of MRC to their patients based on the data of NVC volumes obtained from MRC. For the patient with TN, treatment with CBZ is considered to be the first choice in primary care [32-34]. However, the frequency of side effects from CBZ is considered to be a problem [34-36]. Therefore, a different therapeutic strategy at the early stage may be chosen for the patient in whom an adverse reaction to CBZ is anticipated.

After administration of CBZ at $100 \mathrm{mg} /$ day for 2 weeks as the initial treatment for patients with $\mathrm{TN}$, further treatment with CBZ may be pursued at the physician's discretion based on the patient's symptoms. The package insert for CBZ indicates that the maximum permissible dose is $800 \mathrm{mg} /$ day when used for $\mathrm{TN}$, and should be followed when prescribing CBZ for these patients. Thereafter, it may be determined whether additional treatments are needed at each physician's discretion. That is why no significant correlation was found between the dosage of additional CBZ administered to relieve the pain and the NVC volume on MRC, but not initial administrations of CBZ [8].

\section{CLINICAL SIGNIFICANCE OF MRC FOR THE CURATIVE EFFICACY OF TNB FOR PATIENTS WITH TN}

The flow chart for the treatment of patients with TN at Kyushu Dental College Hospital is shown in Figure 8. Patients with TN who are less likely to have a good re- sult with CBZ can be immediately treated with TNB [14, 15]. In our dental hospital, patients who have benefited less from $\mathrm{CBZ}$ have been also treated by trigeminal nerve block using $0.4 \mathrm{ml}$ TNB at a $5 \%$ concentration as one of the additional therapies. However, the precise factors for predicting the success of TNB remain obscure, and TNB is currently administered at the physician's discretion. Therefore, the relationship between the volume of NVC by MRC and curative effects by TNB was investigated with respect to being predicted by the results of CBZ administration.

To our disappointment, no significant correlation was found between NVC volume on MRC and the success of TNB in patients with TN [13]. On the other hand, the success of TNB might be predicted by the extent of improvement in pain effected by CBZ [13]. It is possible that a patient's response to TNB can be confidently predicted based on the patient's response to CBZ, in addition to simply the physician's judgment based on the patient's symptoms. In addition, it has been found that if TNB were to be effective for a patient, the improvement would be seen within one or two uses. If one administration of TNB does not effectively relieve the pain, up to 5 doses of TNB can be given in total. TNB is a relative dangerous treatment, and the technique's side effects are considered problematic [37]. Therefore, for the patient in whom an adverse reaction to TNB is anticipated, another treatment at an early stage, such as radiotherapy or surgical treatment may be chosen.

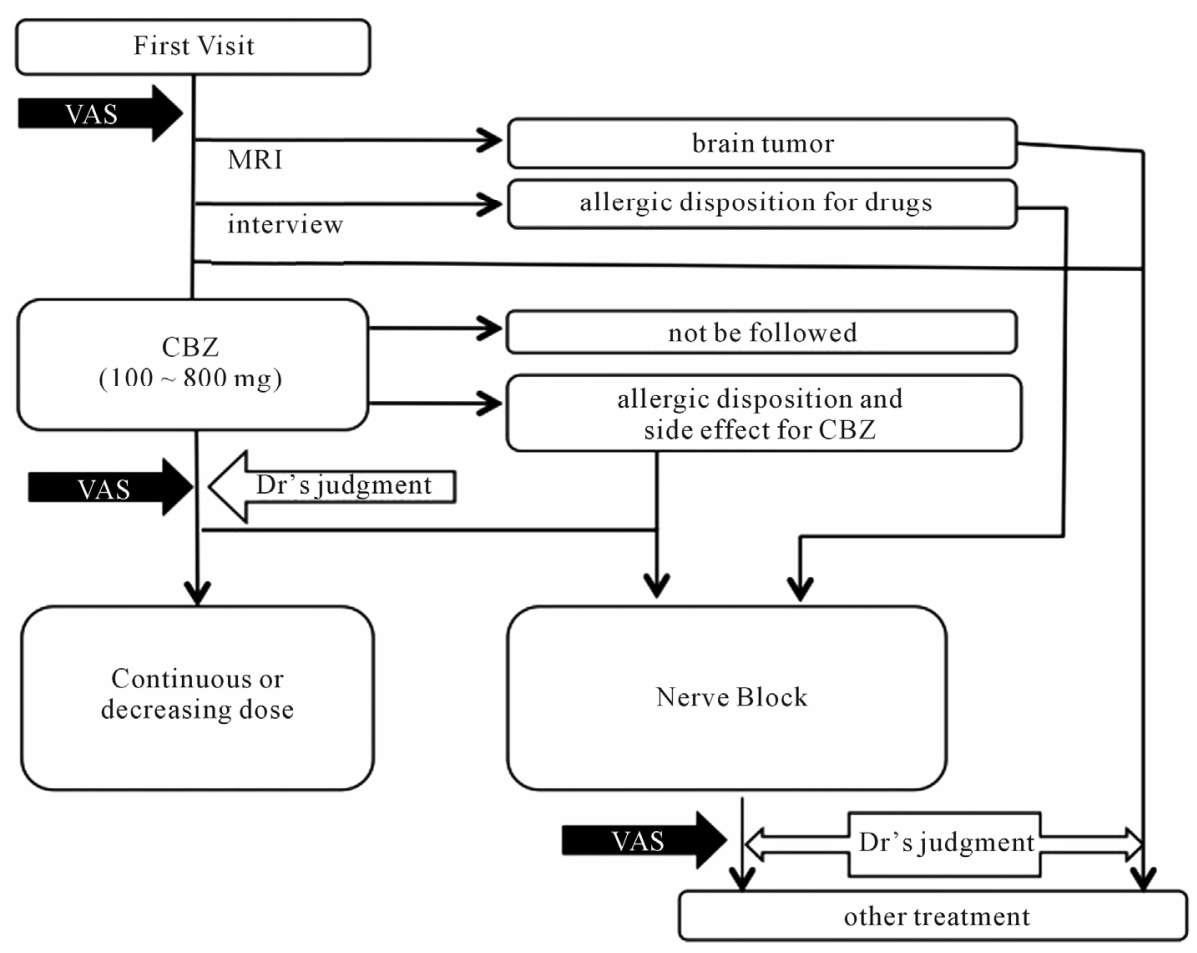

Figure 8. Flow chart of treatment for patients with TN in Kyushu dental college hospital. 


\section{THE FUTURES AND LIMITATIONS ON THE CLINICAL APPLICATIONS OF MRC FOR PATIENTS WITH TN}

Some possible limitations of previous studies were the absence of criteria for deciding the appropriate dosage of CBZ and TNB administration to relieve the pain in patients with TN because the previous studies were retrospective $[7,8,13]$. In addition, CBZ administration was the only initial treatment and there were no criteria for deciding the appropriate TNB. At the same time, the periods for follow-up after treatment were relatively short $[8,13]$. We are planning to evaluate the efficacy of treatments including initial treatments for longer periods and conduct a cohort study as future researches. Other possible limitations were that we could not analyze the relationship between the medications except CBZ and the NVC volumes measured on MRC in patients with TN by NVC [8]. Therefore, as a next research we are planning to analyze the relationship between the medications except CBZ and the NVC volumes measured on MRC in patients with TN by NVC. At the same time we are planning to analyze the relationship between treatments other than TNB, such as radiotherapy and surgical treatments, and the extent of pain or the NVC volumes on MRC in patients with TN caused by NVC.

\section{REFERENCES}

[1] Barker, F.G., Janetta, P.J., Bissonette, D.J., Larkins, M.V. and Jho, H.D. (1996) The long-term outcome of microvascular decompression for trigeminal neuralgia. The New England Journal of Medicine, 334, 1077-1083. doi:10.1056/NEJM199604253341701

[2] Kondo, A. (1997) Follow-up results of microvascular decompression in trigeminal neuralgia and hemifacial spasm. Neurosurgery, 40, 46-51.

[3] Van Loveren, H., Tew, J.M., Keller, J.T. and Nurre, M.A. (1982) A 10-year experience in the treatment of trigeminal neuralgia. Journal of Neurosurgery, 57, 757-764. doi:10.3171/jns.1982.57.6.0757

[4] Boecher-Schwarz, H.G., Bruehl, K., Kessel, G., Guenthner, M., Perneczky, A. and Stoeter, P. (1998) Sensitivity and specificity of MRA in the diagnosis of neurovascular compression in patients with trigeminal neuralgia: A correlation of MRA and surgical findings. Neuroradiology, 40, 88-95. doi:10.1007/s002340050546

[5] Kumon, Y., Sasaki, S., Kohno, K., Ohta, S., Ohue, S. and Miki, H. (1997) Three-dimensional imaging for presentation of the causative vessels in patients with hemifacial spasm and trigeminal neuralgia. Surgical Neurology, 47, 178-184. doi:10.1016/S0090-3019(96)00364-3

[6] Majoie, C.B., Hulsmans, F.J., Verbeeten, Jr., B., Castelijns, J.A., van Beek, E.J., Valk, J. and Bosch, D.A. (1997) Trigeminal neuralgia: Comparison of two MR imaging techniques in the demonstration of neurovascular contact. Radiology, 204, 455-460.
[7] Tanaka, T., Morimoto, Y., Shiiba, S., Sakamoto, E., Kito, S., Matsufuji, Y., Nakanishi, O. and Ohba, T. (2005) Utility of magnetic resonance cisternography using threedimensional fast asymmetric spin-echo sequences with multiplanar reconstruction: The evaluation of sites of neurovascular compression of the trigeminal nerve. Oral Surgery, Oral Medicine, Oral Pathology, Oral Radiology, 100, 215-225.

[8] Tanaka, T., Sakamoto, E., Shiiba, S., Oda, M., Kito, S., Wakasugi-Sato, N., Matsumoto-Takeda, S., Imamura, Y., Nakanishi, O. and Morimoto, Y. (2009) Relationship between the curative effects of carbamazepine administration and neurovascular compression volume of the trigeminal nerve measured using magnetic resonance cisternography. Clinical Journal of Pain, 25, 752-759. doi:10.1097/AJP.0b013e3181ada29c

[9] Yoshino, N., Akimoto, H., Yamada, I., Nagaoka, T., Tetsumura, A., Kurabayashi, T., Honda, E., Nakamura, S. and Sasaki, T. (2003) Trigeminal neuralgia: Evaluation of neuralgic manifestation and site of neurovascular compression with 3D CISS MR imaging and MR angiography. Radiology, 228, 539-545. doi:10.1148/radiol.2282020439

[10] Akimoto, H., Nagaoka, T., Nariai, T., Takada, Y., Ohno, K. and Yoshino, N. (2002) Preoperative evaluation of neurovascular compression in patients with trigeminal neuralgia by use of three-dimensional reconstruction from two types of high-resolution magnetic resonance imaging. Neurosurgery, 51, 956-961.

[11] Yamakami, I., Kobayashi, E., Hirai, S. and Yamaura, A. (2000) Preoperative assessment of trigeminal neuralgia and hemifacial spasm using constructive interference in steady state-three-dimensional Fourier transformation magnetic resonance imaging. Neurologia Medico-Chirurgica, 40, 545-555. doi:10.2176/nmc.40.545

[12] Schmitz, S.A., Hohenbleicher, H., Koennecke, H.C., Offermann, R., Offermann, J., Branding, G., Wolf, K.J., Distler, A. and Sharma, A.M. (1999) Neurogenic hypertension. A new MRI protocol for the evaluation of neurovascular compression of the cranial nerves IX and $\mathrm{X}$ root-entry zone. Investigative Radiology, 34, 774-780. doi:10.1097/00004424-199912000-00007

[13] Shiiba, S., Tanaka, T., Sakamoto, E., Oda, M., Kito, S., Ono, K., Wakasugi-Sato, N., Matsumoto-Takeda, S., Seta, Y., Imamura, Y., Nakanishi, O., Inenaga, K. and Morimoto, Y. (2012) Can the neurovascular compression volume of the trigeminal nerve on MR cisternography predict the success of local anesthetic block after initial treatment by the carbamazepine? Oral Surgery, Oral Medicine, Oral Pathology, Oral Radiology, in press.

[14] Radwan, I.A., Saito, S. and Goto, F. (2001) High-concentration tetracaine for the management of trigeminal neuralgia: Quantitative assessment of sensory function after peripheral nerve block. Clinical Journal of Pain, 17, 323-326. doi:10.1097/00002508-200112000-00006

[15] Goto, F., Ishizaki, K., Yoshikawa, D., Obata, H., Arii, H. and Terada, M. (1999) The long lasting effects of peripheral nerve blocks for trigeminal neuralgia using high concentration of tetracaine dissolved in bupivacaine. Pain, 79, 101-103. doi:10.1016/S0304-3959(98)00156-0 
[16] Naganawa, S., Koshikawa, T., Fukatsu, H., Ishigaki, T. and Fukuta, T. (2001) MR cisternography of the cerebellopontine angle: Comparison of three-dimensional fast asymmetrical spin-echo and three-dimensional constructive interference in the steady-state sequences. American Journal of Neuroradiology, 22, 1179-1185.

[17] Tsuchiya, K., Yamakami, N., Hachiya, J. and Kassai, Y. (1998) MR cisternography using a three-dimensional half-Fourier single-shot fast spin-echo sequence. European Radiology, 8, 424-426. doi:10.1007/s003300050405

[18] Nakamura, T., Naganawa, S., Koshikawa, T., Fukatsu, H., Sakurai, Y., Aoki, I., Ninomiya, A. and Ishigaki, T. (2002) High-spatial-resolution MR cisternography of the cerebellopontine angle in 90 seconds with a zero-fill interpolated fast recovery 3D fast asymmetric spin-echo sequence. American Journal of Neuroradiology, 23, 14071412.

[19] Tsunoda, A., Mitsuoka, H., Sato, K. and Kanayama, S. (2000) A quantitative index of intracranial cerebrospinal fluid distribution in normal pressure hydrocephalus using an MRI-based processing technique. Neuroradiology, 42, 424-429. doi:10.1007/s002349900241

[20] Morimoto, Y., Tanaka, T., Yoshioka, I., Masumi, S., Yamashita, M. and Ohba, T. (2002) Virtual endoscopic view of salivary gland ducts using MR sialography data from three-dimensional fast asymmetric spin-echo (3D-FASE) sequences: A preliminary study. Oral Diseases, 8, 268274. doi:10.1034/j.1601-0825.2002.01819.X

[21] Morimoto, Y., Tanaka, T., Tominaga, K., Yoshioka, I., Kito, S. and Ohba, T. (2004) Clinical application of MR sialographic 3D-reconstruction imaging and MR virtual endoscopy for salivary gland duct analysis. Journal of Oral and Maxillofacial Surgery, 62, 1237-1245. doi:10.1016/j.joms.2003.12.035

[22] Adamek, H.E., Albert, J., Breer, H., Weitz, M., Schilling, D. and Riemann, J.F. (2000) Pancreatic cancer detection with magnetic resonance cholangiopancreatography and endoscopic retrograde cholangiopancreatography: A prospective controlled study. Lancet, 356, 190-193. doi:10.1016/S0140-6736(00)02479-X

[23] Neri, E., Boraschi, P., Braccini, G., Caramella, D., Perri, G. and Bartolozzi, C. (1999) MR virtual endoscopy of pancreaticobiliary tract. Magnetic Resonance Imaging, 17, 59-67. doi:10.1016/S0730-725X(98)00127-1

[24] Yamakawa, K., Naganawa, S., Maruyama, K., Kato, T., Fukatsu, H. and Ishigaki, T. (1999) Clinical evaluation of three-dimensional MR-cholangiopancreatography using three-dimensional Fourier transform fast asymmetric spin echo method (3DFT-FASE): Usefulness of observation by multiplanar reconstruction. Radiation Medicine, 17, 1519.

[25] El Gamma, T. and Brooks, B.S. (1994) MR cisternography: Initial experience in 41 cases. American Journal of Neuroradiology, 15, 1647-1656.

[26] El Gammal, T., Sobol, W., Wadlington, V.R., Sillers, M.J., Crews, C., Fisher, III, W.S. and Lee, J.Y. (1998) Cerebrospinal fluid fistula: Detection with MR cister- nography. American Journal of Neuroradiology, 19, $627-$ 631.

[27] Mamata, Y., Muro, I., Matsumae, M., Komiya, T., Toyama, H., Tsugane, R. and Sato, O. (1998) Magnetic resonance cisternography for visualization of intracranial fine structures. Journal of Neurosurgery, 88, 670-678. doi:10.3171/jns.1998.88.4.0670

[28] Worthington, C., Hutson, K., Boulware, R., Neglia, W., Gibbons, J.P., Clark, R. and Rand, J. (2000) Computerized tomography cisternography of the trigeminal nerve for stereotactic radiosurgery. Case report. Journal of Neurosurgery, 93, 169-171.

[29] Gudmundsson, K., Rhoton, Jr., A.L. and Rushton, J.G. (1971) Detailed anatomy of the intracranial portion of the trigeminal nerve. Journal of Neurosurgery, 35, 592-600. doi:10.3171/jns.1971.35.5.0592

[30] Sindou, M., Leston, J., Decullier, E. and Chapuis, F. (2007) Microvascular decompression for primary trigeminal neuralgia: Long-term effectiveness and prognostic factors in a series of 362 consecutive patients with clear-cut neurovascular conflicts who underwent pure decompression. Journal of Neurosurgery, 107, 1144-1153. doi:10.3171/JNS-07/12/1144

[31] Sindou, M., Howeidy, T. and Acevedo, G. (2002) Anatomical observations during microvascular decompression for idiopathic trigeminal neuralgia (with correlations between topography of pain and site of the neurovascular conflict). Prospective study in a series of 579 patients. Acta Neurochirurgica, 144, 1-12. doi:10.1007/s701-002-8269-4

[32] Cruccu, G., Gronseth, G., Alksne, J., Argoff, C., Brainin, M., Burchiel, K., Nurmikko, T. and Zakrzewska, J.M. (2008) American Academy of Neurology Society; European Federation of Neurological Society. AAN-EFNS guidelines on trigeminal neuralgia management. European Journal of Neurology, 15, 1013-1028. doi:10.1111/j.1468-1331.2008.02185.x

[33] Jorns, T.P. and Zakrzewska, J.M. (2007) Evidence-based approach to the medical management of trigeminal neuralgia. British Journal of Neurosurgery, 21, 253-261. doi:10.1080/02688690701219175

[34] Hvidberg, E.F. and Dam, M. (1976) Clinical pharmacokinetics of anticonvulsants. Clinical Pharmacokinetics, 1, 161-188. doi:10.2165/00003088-197601030-00001

[35] Bertilsson, L. (1978) Clinical pharmacokinetics of carbamazepine. Clinical Pharmacokinetics, 3, 128-143. doi:10.2165/00003088-197803020-00003

[36] Rozen, T.D. (2001) Antiepileptic drugs in the management of cluster headache and trigeminal neuralgia. Headache, 41, S25-S32. doi:10.1046/j.1526-4610.2001.01154-5.x

[37] Peters, G. and Nurmikko, T.J. (2002) Peripheral and gasserian ganglion-level procedures for the treatment of trigeminal neuralgia. Clinical Journal of Pain, 18, 28-34. doi:10.1097/00002508-200201000-00005 